\title{
Metagenomic monitoring of soil bacterial community after the construction of a crude oil flowline
}

\author{
Maria Grazia Bonomo (D) Luana Calabrone $\cdot$ Laura Scrano • \\ Sabino Aurelio Bufo • Katia Di Tomaso • Euro Buongarzone • \\ Giovanni Salzano
}

Received: 6 May 2021 / Accepted: 20 November 2021 / Published online: 3 January 2022

(C) The Author(s) 2022

\begin{abstract}
This study aimed to assess the metagenomic changes of soil bacterial community after constructing a crude oil flowline in Basilicata region, Italy. Soils identified a total of 56 taxa at the phylum level and 485 at the family level, with a different taxa distribution, especially in samples collected on 2014. Since microbiological diversity occurred in the soils collected after 2013 (the reference year), we performed a differential abundance analysis using DESeq 2 by GAIA pipeline. In the forest area, 14 phyla and 126 families were differentially abundant $(-6.06<\log \mathrm{FC}>7.88)$ in 2014 compared to 2013. Nine families were differentially abundant in 2015,
\end{abstract}

Supplementary Information The online version contains supplementary material available at https://doi. org/10.1007/s10661-021-09637-3.

M. G. Bonomo $(\bowtie) \cdot$ L. Calabrone · S. A. Bufo ·

K. Di Tomaso $\cdot$ G. Salzano

Department of Sciences, University of Basilicata, Potenza, Italy

e-mail: mariagrazia.bonomo@unibas.it

L. Scrano

Department of European Cultures, University

of Basilicata, Potenza, Italy

S. A. Bufo

Department of Geography, Environmental Management and Energy Studies, University of Johannesburg,

Johannesburg, South Africa

E. Buongarzone

SAIPEM S.P.A, Fano, Italy with $\log F C$ between -3.16 and 4.66 , while 20 families were significantly more abundant and 16 less abundant in 2016, with $\log \mathrm{FC}$ between -6.48 and 6.45 . In the cultivated area, 33 phyla and 260 families showed differential abundance in 2014. In the next year (2015), 14 phyla were significantly more abundant and 19 less abundant, while 29 families were substantially more abundant and 139 less abundant, with fold changes ranging between -5.67 and 4.01. In 2016, 33 phyla showed a significantly different abundance, as 14 were more abundant and 19 decreased, and 81 families showed a significantly increased amount with $\log \mathrm{FC}$ between -5.31 and 5.38. These results hypothesise that the analysed site is an altered soil where the development of particular bacterial groups attends to bioremediation processes, naturally occurring to restore optimal conditions.

Keywords Soil bacterial diversity $\cdot$ Microbial composition changes $\cdot$ Metagenomic analyses $\cdot$ Oil flowline effects

\section{Introduction}

Soil is a rich and dynamic ecosystem representing the most vast microbial diversity source on the entire world. This hidden biodiversity could be a great resource of natural products for agriculture and biotechnological applications (Mocali \& Benedetti, 2010; Ahmed et al., 2018). 
Soil microorganisms play a crucial role in ecologically critical biogeochemical processes, contributing to plant nutrition and soil health, even in agricultural and extreme environments, and maintaining the matter and energy transfer in terrestrial environments (Arias et al., 2005; Lelario et al., 2018; Mader et al., 2002; Sofo et al., 2018). Soil represents, with its composite microbial functions, a very complex and heterogeneous environment for microbiologists. Due to soil properties and interaction processes, involving mineral and organic particles, soil biota gives rise to the formation and stabilisation of differently sized aggregates, micropores and clay-organic matter complexes that dominate the soil characteristics and affect the microbial composition (Mocali \& Benedetti, 2010).

Besides, microbial communities in soil are randomly spread out, following the best growing conditions, such as nutrient gradients and moisture content, and leading to the so-called hot-spot distribution (Mocali \& Benedetti, 2010). Most soil microbial communities represent a new source of genetic and metabolic diversity; in fact, only a small fraction of the soil bacteria (less than 1\%) is cultivable, highlighting the need to assess and preserve the diversity in soil microorganisms' distribution (Mocali \& Benedetti, 2010).

Traditional microbiological approaches present severe limitations to knowledge of soil microbial diversity (Mocali \& Benedetti, 2010; Torsvik \& Ovreas, 2002). Therefore, in the last decades, molecular fingerprinting techniques were developed, representing a rapid and powerful tool for understanding soil microbial communities' dynamics and diversity (Bonomo \& Salzano, 2013; Bonomo et al., 2013; van Elsas et al., 2007). However, these approaches proved limitations and biases, mainly related to the target gene's characteristics and PCR amplification efficiency, which have always limited knowledge to a restricted part of the microbial communities (Kirk et al., 2004; Cafaro et al., 2016). For these reasons, novel approaches to exploring the vast majority of soil microbial diversity were necessary (Mocali \& Benedetti, 2010).

In recent years, several molecular approaches have been proposed (van Elsas et al., 2007; Kirk et al., 2004; Bloem et al., 2006; Bonomo et al., 2017; Sorensen et al., 2009) and, recently, the exploration of entire genomes present in a soil sample, metagenomics, has provided a new approach for detailed assessment (Mocali \& Benedetti, 2010; Daniel, 2005; Schloss \& Handelman, 2005). Metagenomics is a powerful tool for studying the soil molecular ecology, assessing the diversity of complex microbial communities, providing access to several new species, genes or novel molecules relevant for biotechnology and agricultural applications (Mocali \& Benedetti, 2010).

The metagenomic approach allows to obtain useful information on the composition and geneticphysiological mechanisms of soil microbiota and their adaptation to specific environments, such as oilcontaminated soils, for a better understanding of the alterations of microbial development and biochemical activities and bioremediation processes (Mocali \& Benedetti, 2010; Ahmed et al., 2018; Gomez et al., 2004; Peng et al., 2015) fundamental to re-establish soil microbial communities (Rutgers et al., 2016; Galazka et al., 2018).

Recent advances in genomics, transcriptomics and proteomics have led to increased studies on bacterial communities in contaminated soil. Genomic methods include functional bacterial fingerprinting and nextgeneration sequencing (NGS) of hypervariable regions, such as in 16S rRNA genes from bacteria, to determine the genetic diversity of microorganisms within a population without the need for cell culture (Galazka et al., 2018; Malla et al., 2018; Pichler et al., 2018).

In this study, different soil samples, taken ante- and post-opera the installation of an oil pipeline, were subjected to analysis of NGS to study and investigate the complex microbial biodiversity. Due to the variety of chemical-metabolic processes involved, the biodiversity of soil microorganisms plays an essential role in maintaining ecosystems in a functionally efficient state.

This study monitors the changes in the soil bacterial community's composition in the 3 years following the construction of a crude oil flowline. The objective is to verify the possible recovery and the restoration over 3 years of the analysed territory's initial conditions and its reuse for crops and leisure areas.

The evaluation of the displacements of the composition of the bacterial community of the soil is essential for understanding and deepening the activities and microbial dynamics in the soil, i.e. the relationships between functionality and microbial diversity, at the basis of the fundamental recovery and remediation processes. Furthermore, the aim is to identify 
the predominant taxa that can act as model organisms and microbial indicators of soil stress induced by the flowline construction works.

\section{Materials and methods}

Site description and sampling

A farmhouse located in the Basilicata region (South Italy), affected by the flowline construction works, has been the study's sampling site. The crude oil flowline consisted of a 4-m wide excavation, $4 \mathrm{~m}$ deep and about $1500 \mathrm{~m}$ long. The flow of earthmoving vehicles involved a 40-m strip around the excavation. A forest area and a cultivated area (divided between arable land and tree crops) characterise the concerned territory's subdivision. Figure S1 shows sampling points of the considered area. The monitoring started with soil samples collected in June and July 2013 (ante-opera monitoring) and continued during the 3 years after the flowline construction (post-opera monitoring).

Four sampling points occurred in the forest area and five in the cultivated area to assess the specific features and dynamics of microbial ecology and their evolution induced by environmental restoration processes. The monitoring program provided $2 \mathrm{~kg}$ of soil collected at a depth of $0-20 \mathrm{~cm}$ and $20-40 \mathrm{~cm}$ from all sampling points. A part of the sample kept in a thin layer at ambient temperature in a plastic tray was in use for physical-chemical analyses (detailed methods used and obtained data are available in Supplementary Material). The remaining part frozen at $-20{ }^{\circ} \mathrm{C}$ was useful for the subsequent DNA extraction and culture-independent microbial diversity analyses.

16S rDNA amplicon sequencing and sequence processing

According to the manufacturer's protocol, soil DNA extraction was from $20 \mathrm{~g}$ of each soil sample, using ZR Soil Microbe DNA MicroPrep ${ }^{\mathrm{TM}}$ Kit (Zymo Research, Italy). The isolated soil DNA visualisation was possible by agarose gel $(1.0 \%$, w/v) electrophoresis running, and its quantification using Nanodrop Spectrophotometer ND-1000 (Thermo Fisher Scientific, Italy). Microbial genomic DNA extraction was in triplicate for each sample.
The extract DNA used to prepare 16S gene amplicon libraries at IGAtechnology (Udine, Italy) was according to the Illumina protocol (C) 16S Metagenomic Sequencing Library Preparation protocol and aiming at sequencing the $\mathrm{V} 3$ and $\mathrm{V} 4$ variable regions with primers 16S-341F 5'-CCTACGGGNGGCWGCAG-3' and 16S-805R 5'-GACTACHVGGGTATCTAATCC-3'.

A subsequent amplification was necessary to index sequences on the sequencing cell (NexteraXT Index Kit, FC-131-1001/FC-131-1002). Finally, the libraries were sequenced in the MiSeq Illumina platform to obtain 300 bp paired reads.

At the end of the sequencing, we evaluated the reads' quality through FASTQC. A processing was compulsory to eliminate the sequencing primers and the reads of low quality using the Trimmomatic program. An additional quality control with FASTQC was necessary.

\section{Bioinformatics analysis}

For the metagenomic analysis and differential analysis, the GAIA pipeline was used, developed by Sequentia Biotech SL (Barcelona, Spain). This pipeline uses high quality reads to map them with BWA against the NCBI database to identify the taxonomy it belongs to and uses the low common ancestor (LCA) algorithm to classify them. Identity thresholds are applied to classify reads into operational taxonomic units (OTUs) at species, genus, family, phylum and domain levels.

LCA algorithm allowed the identification to the following percentages: identity between 0 and $70 \%$ : reads assigned at the domain level; identity between 71 and $73 \%$ : reads displayed at the phylum level; identity between 74 and $85 \%$ : reads posted at family level; correspondence between 86 and 93\%: reads allocated to the genus level; and identity between 94 and $97 \%$ : reads assigned at the species level; the reads mapped to a unique species were also classified in the species reference strain to which they mapped, while the reads that did not map appeared in the report as unknown.

Each identified taxa could estimate the absolute abundance (reported as number of reads/pair counts) and relative abundance (reported as a percentage). The data reported as bar plots detect taxa present in each sampling point and their relative abundance. For convenience, taxa with less than $0.1 \%$ abundance in all samples were grouped as 


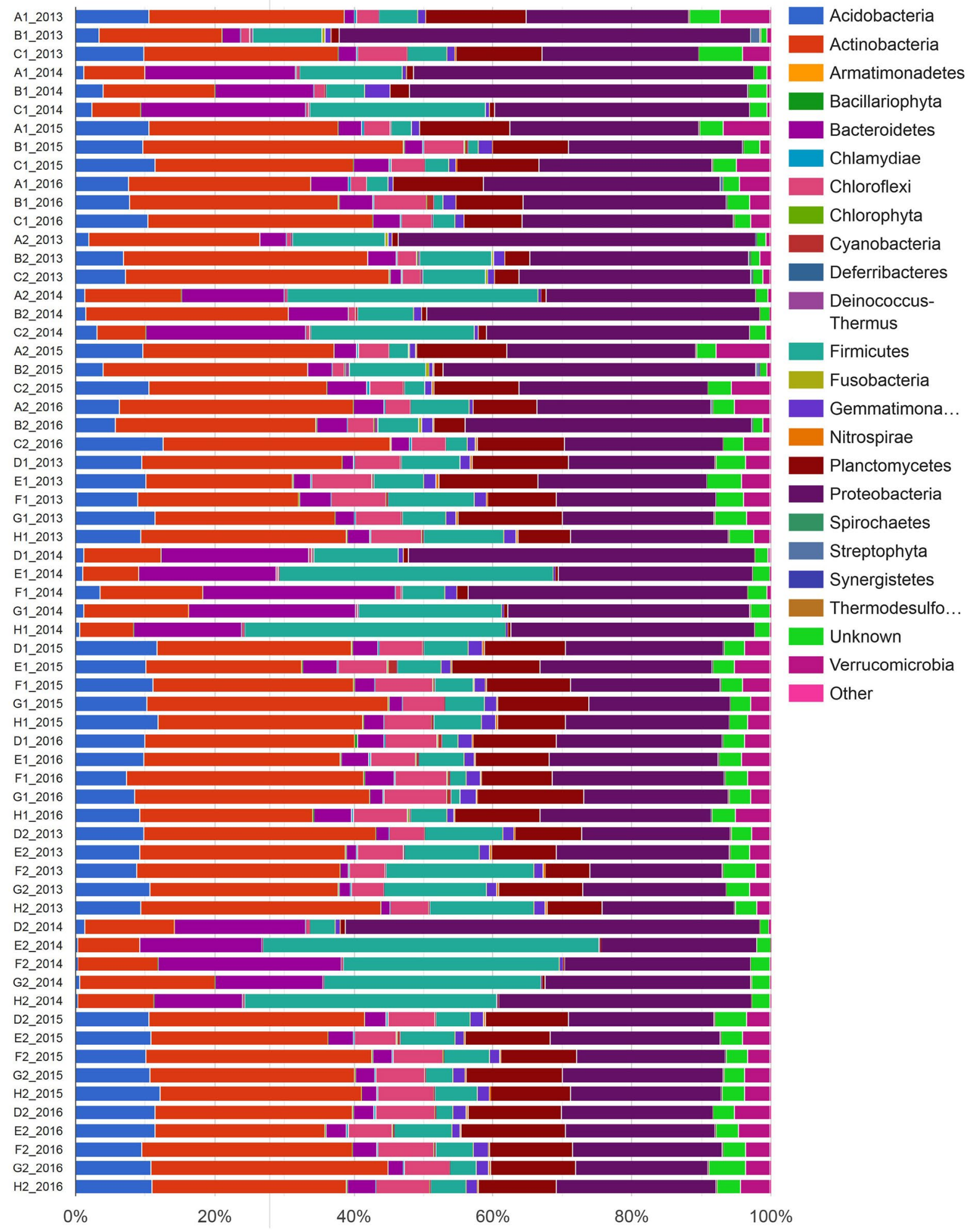


4Fig. 1 Taxa distribution in soil samples at the taxonomic level of phylum. The samples are sorted by sampling area (forest: $\mathrm{ABC}$, cultivated: DEFGH), by year (following the chronological order of sampling) and by depth, showing for each group the sampling layer 0-20 cm (1) and then the layer 20-40 cm (2)

others. The samples are sorted by sampling area (forest: $\mathrm{ABC}$, cultivated: DEFGH), by year (following the chronological order of sampling) and by depth, showing for each group the sampled points at $0-20 \mathrm{~cm} \mathrm{(1)} \mathrm{and} \mathrm{then} \mathrm{those} \mathrm{at} \mathrm{20-40} \mathrm{cm} \mathrm{(2).}$

Alpha and beta diversities, calculated using Phyloseq in $\mathrm{R}$, indicate the richness level (taxa number) and evenness level (taxa relative abundance) in the different soil samples. They also calculate the distance between the pairs of samples as a matrix of dissimilarity of Bray-Curtis.

Moreover, a differential abundance analysis using DESeq2, performed by GAIA, identify taxa differentially abundant in samples grouped by area and year of sampling and compared as follows: forest area 2014 vs forest area 2013, forest area in 2015 vs forest area in 2013, forest area 2016 vs forest area 2013, cultivated area 2014 vs cultivated area 2013, cultivated area 2015 vs cultivated area 2013 and cultivated area 2016 vs cultivated area 2013.

We standardised the counts resulting from the mapping of the reads to the reference database to compare different samples. Principal component analysis (PCA) was useful for studying the variation within the groups of compared samples and observing their distribution according to the relative distance.

We believed useful to construct a Volcano and an MA diagram to represent the estimated differential abundance levels.

Finally, we analysed the number of taxa for each taxonomic level as more (over-represented) or less (under-represented) abundant compared to the reference year (2013). We described their abundance as the decimal $\log$ of the fold change (logFC, i.e. the number of times the abundance has changed significantly compared to the base year 2013).

\section{Results and discussion}

The soil disturbance created by the excavation, movement of heavy vehicles and destruction of the natural horizons of the soil and artificial reconstruction of layers, which cannot reproduce exactly the initial situation, determined a mixing of the horizons with modification of the characteristics along the soil profile. In this study, different soil samples, taken ante- and post-construction of a crude oil flowline, were analysed by next-generation sequencing (NGS) to investigate the complex microbial biodiversity that plays a vital role in maintaining ecosystems in a functionally efficient state.

In this study, we identified a total of 56 taxa at the phylum level, 485 at the family level, 1190 at the genus level and 23,232 at the species level. Soil samples showed a different taxa distribution, especially in each of the groups collected in 2014. Considering the taxonomic level of the phylum, in Fig. 1, the overall most abundant and variable taxa appear to be Acidobacteria and Actinobacteria, which are less abundant in 2014; Bacteroidetes, which increased significantly in 2014; Chloroflexi; Firmicutes, particularly abundant on the site labelled $\mathrm{C}$ and agricultural soils in 2014; and Proteobacteria and Verrucomicrobia, apparently absent in the 2014 agricultural samples.

Moreover, regarding the family taxonomic level in Fig. 2, the abundant and variable taxa are Acidobacteriaceae, Bacillaceae, Hymenobacteriaceae, Micrococcaceae, Oxalobacteriaceae, Paenibacillaceae, Planctomycetaceae, Pseudomonadaceae, Propionibacteriaceae and Rubrobacteriaceae.

The heatmaps, built for easy and immediate visualisation of data, presented the best results at the taxonomic levels of phylum and family. Figures 3 and 4 clearly show the abundance blocks of different taxa in sampling sites during the three years following the flowline's construction, suggesting changes, i.e. biodiversity.

The results obtained agree with previous works that showed differences in soil microbial community structure and taxonomic composition due to different anthropic insults (Ahmed et al., 2018; Galazka et al., 2018; Carbonetto et al., 2014; Liu et al., 2017; Klimek et al., 2016). We observed significant alterations of microbiological dynamics following the flowline construction compared to the reference year 2013 (ante-opera monitoring) suggesting their evolution induced by environmental restoration processes. Also, changes were detected in the post-opera years, highlighting a specific adaptation to the altered conditions by select groups and types of bacteria. Changes in bacterial communities observed in post-opera analyses 


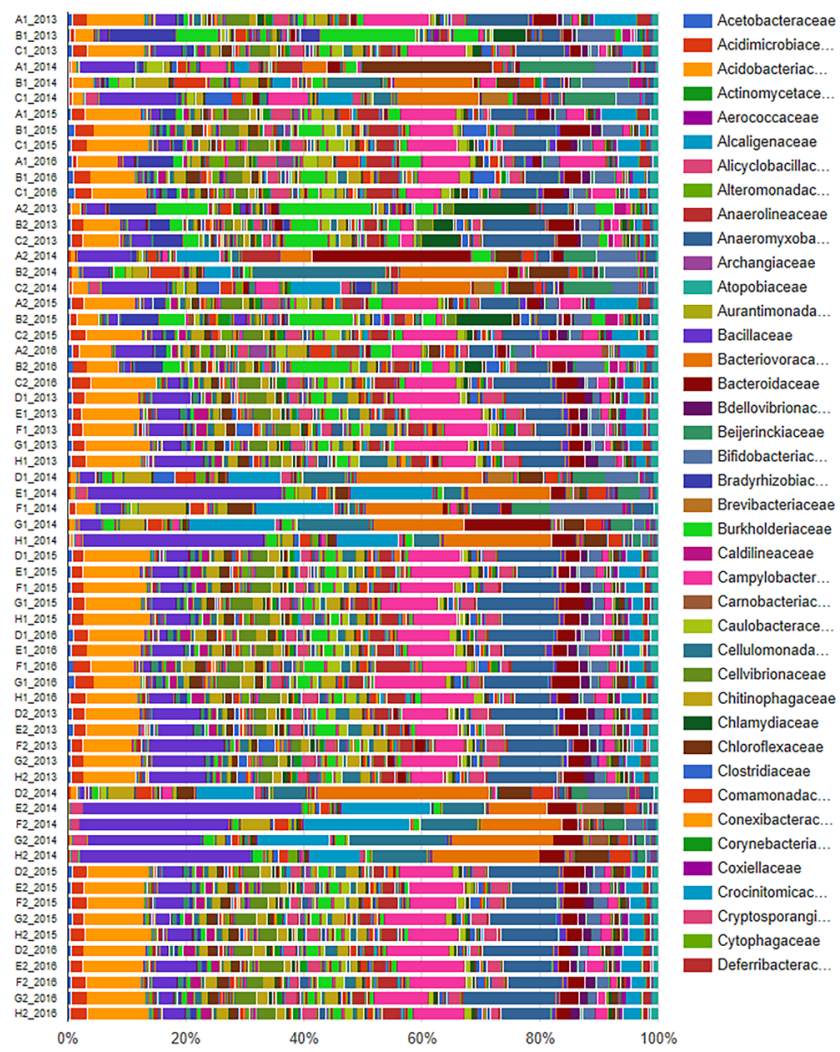

Fig. 2 Taxa distribution in soil samples at the taxonomic level of family. The samples are sorted by sampling area (forest: $\mathrm{ABC}$, cultivated: DEFGH), by year (following the chronologi-

demonstrated modifications of the genetic and physiological bacterial response to face stress. Bacterial communities implemented different mechanisms to survive and adapt to the modified conditions of the soil environment.

As shown in Tables $\mathrm{S} 1$ and $\mathrm{S} 2$, the extensive soil disturbance due to the flowline construction inevitably caused direct and indirect changes in soil characteristics. The forest area has undergone a substantial evolution with a noteworthy diversification of chemical-physical parameters between the different sampling points. The chemical-physical properties of the examined soils were initially almost uniform but showed significant annual variations. The phenomenon indicates aggregation processes favouring organo-mineral associations in an environment with good microbial biomass activity that can suggest the development of widespread root systems.
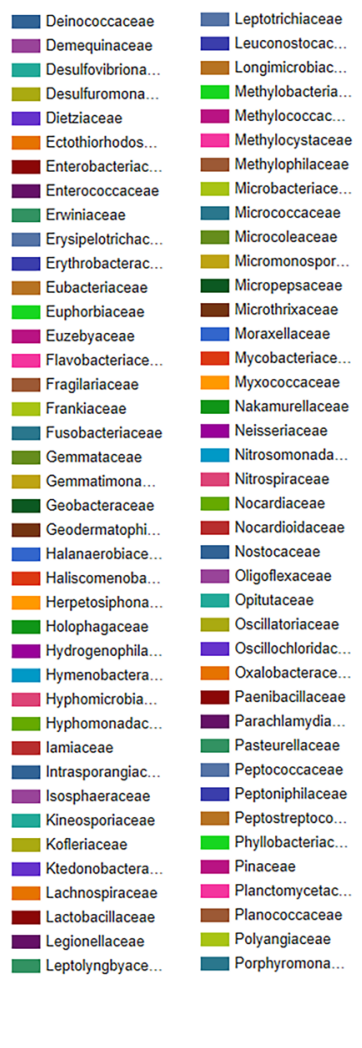

Verrucomicrobi...

$\square$ Vibrionaceae

Williamsiaceae

Xanthobacterac.

Xanthomonada

$\square$ Yersiniaceae

Other

Prich

Rhodanobacter

Rhodobacterac.

Rhodobiaceae

Rhodocyclaceae

Rhodospirillaceae

- Rikenellaceae

Roseiflexaceae

Rubrobacterac..

Ruminococcac.

Saprospiraceae

- Selenomonada

Sinobacteracea

$\square$ Solanaceae

Solirubrobacter

Sphaerobacter.

Sphingobacteri.

Sphingomonad..

Spirochaetacea

Sporomusaceae

Staphylococcac

Streptococcaceae

Streptomycetac.

Streptosporang

- Synergistaceae

Thermaceae

Thermoactinom.

Thermoanaero

Thermoleophila.

Thermomicrobi.

Thermomonosp.

Thiotrichaceae

Tissierellaceae

- Veillonellaceae

Verrucomicrobi. cal order of sampling) and by depth, showing for each group the sampling layer $0-20 \mathrm{~cm}$ (1) and then the layer $20-40 \mathrm{~cm}$ (2)

The $\mathrm{pH}$ values tend to evolve towards greater alkalinity, which is also identifiable in the CEC values that are all higher than the initial ones, even when reducing the free organic matter.

We hypothesise that, in addition to the climatic factors and rainfall, always abundant in the area (data not shown), the soil's movement first, and the development of grassy vegetation then, played a fundamental role in the observed evolutionary process. The reduction of organic carbon is well observable together with a similar decrease in total nitrogen, exchangeable potassium and assimilable phosphorus.

The values of concentrations found for the heavy hydrocarbons (from $\mathrm{C} 10$ to $\mathrm{C} 40$ ) were generally under the limit of detection attributable to the chromatographic method used $(5 \mathrm{mg} / \mathrm{kg}$ of dry matter). In 2014, for both the forest area and the cultivated area, values unusually higher of hydrocarbons were 


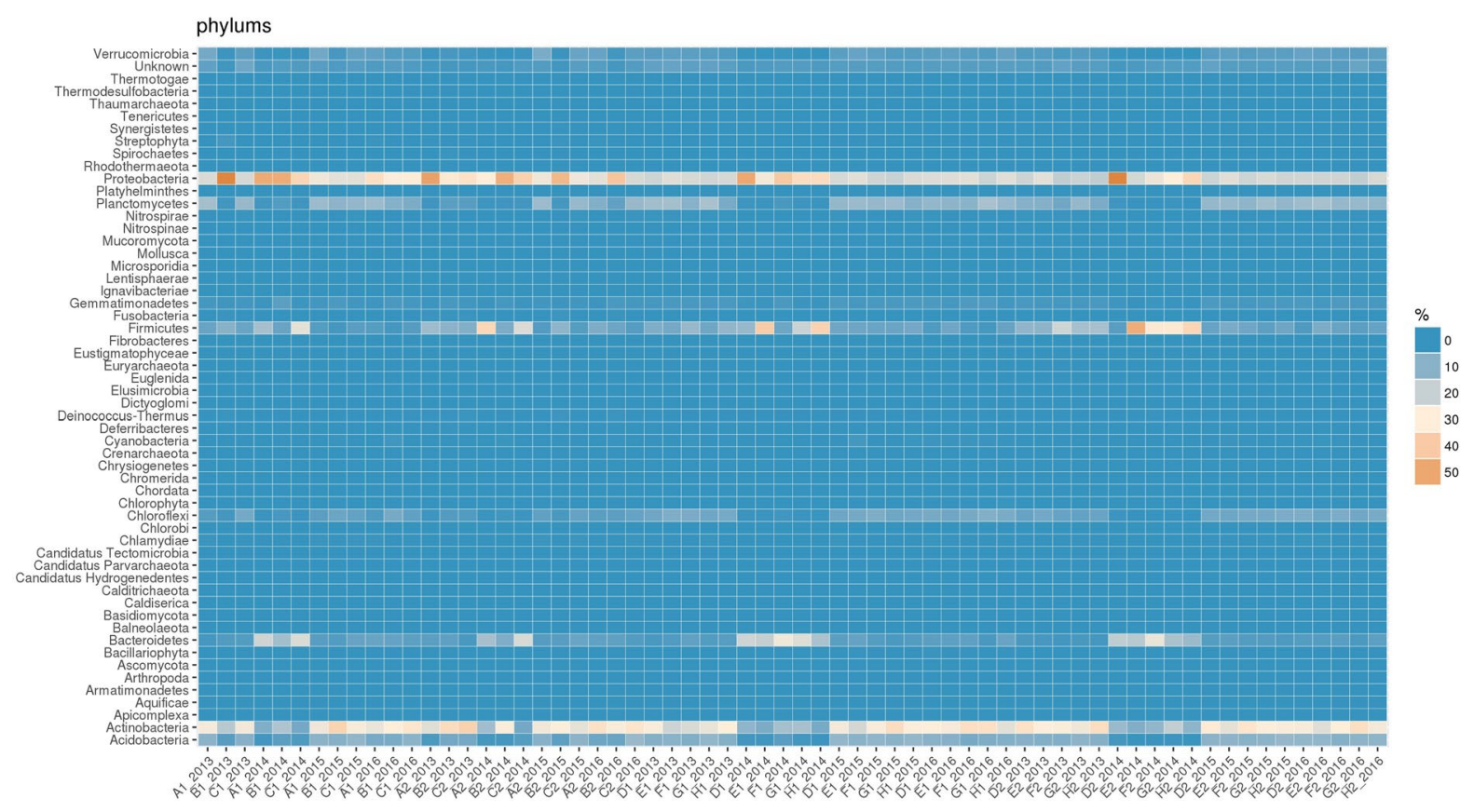

Fig. 3 Heatmap for data visualisation at the taxonomic level of phylum; abundance blocks of different taxa in the sampling sites during the 3 years following the flowline's construction

recorded in either the superficial- and deep-sampled soil layers (16-32 mg/kg dm). This event suggests a small and momentary leak in the oil pipeline that was operative at the end of 2013. In 2015, a weird value appeared only in a deep sample collected from the agricultural area $(36 \mathrm{mg} / \mathrm{kg} \mathrm{dm})$. Such a recovery process occurs only when different microbial species can transform the hydrocarbons into energy, attending a natural bioremediation/biodegradation activity and restoring the initial soil conditions.

Since microbiological diversity changes occurred in the samples collected after the year 2013, a comparative analysis was necessary to identify taxonomies whose abundance is significantly changing over time. Therefore, we performed a differential abundance analysis using DESeq2 by GAIA to identify taxa differentially represented.

We evaluated the abundance of taxa by comparing the forest area sampling in 2014 and the selection of 2013. The Principal component analysis in Fig. S2a showed a variability of the 2014 samples more significantly than the 2013 samples at the phylum taxonomic level, explaining the new biological differentiation between the two sampling years. The analysis of the differences groups the principal component
1 (X-axis) quite differently over the 2 years, while the 2013 samples clustered in a more homogeneous group. The main component 1 explains $75 \%$ of the total variation. The method adopted recognised 56 phyla in this group of samples; 14 of these are differentially abundant in 2014 compared to the reference year (2013). In particular, eight phyla seem overrepresented, i.e. more abundant in 2014 than 2013, and 6 under-represented, i.e. they showed a lower abundance in 2014.

At the family taxonomic level, the PCA showed an even more significant variability among the 2014 samples than those of 2013 regarding biological diversity. The main component 2 , accounting for $22 \%$ variability, collects most of the diversity of the 2014 collected soils, except for site A at a depth of 20-40 m, which is very far from the others along the X-axis in Fig. S2b. This group of samples identify 485 taxonomic families. The comparative analysis highlighted that 126 families are differentially present in 2014 compared to 2013. In particular, 65 increased quantitatively, and 59 significantly decreased in 2014. Table 1 shows the significant taxa under and over-represented, with the relative value of $\log \mathrm{FC}$. 


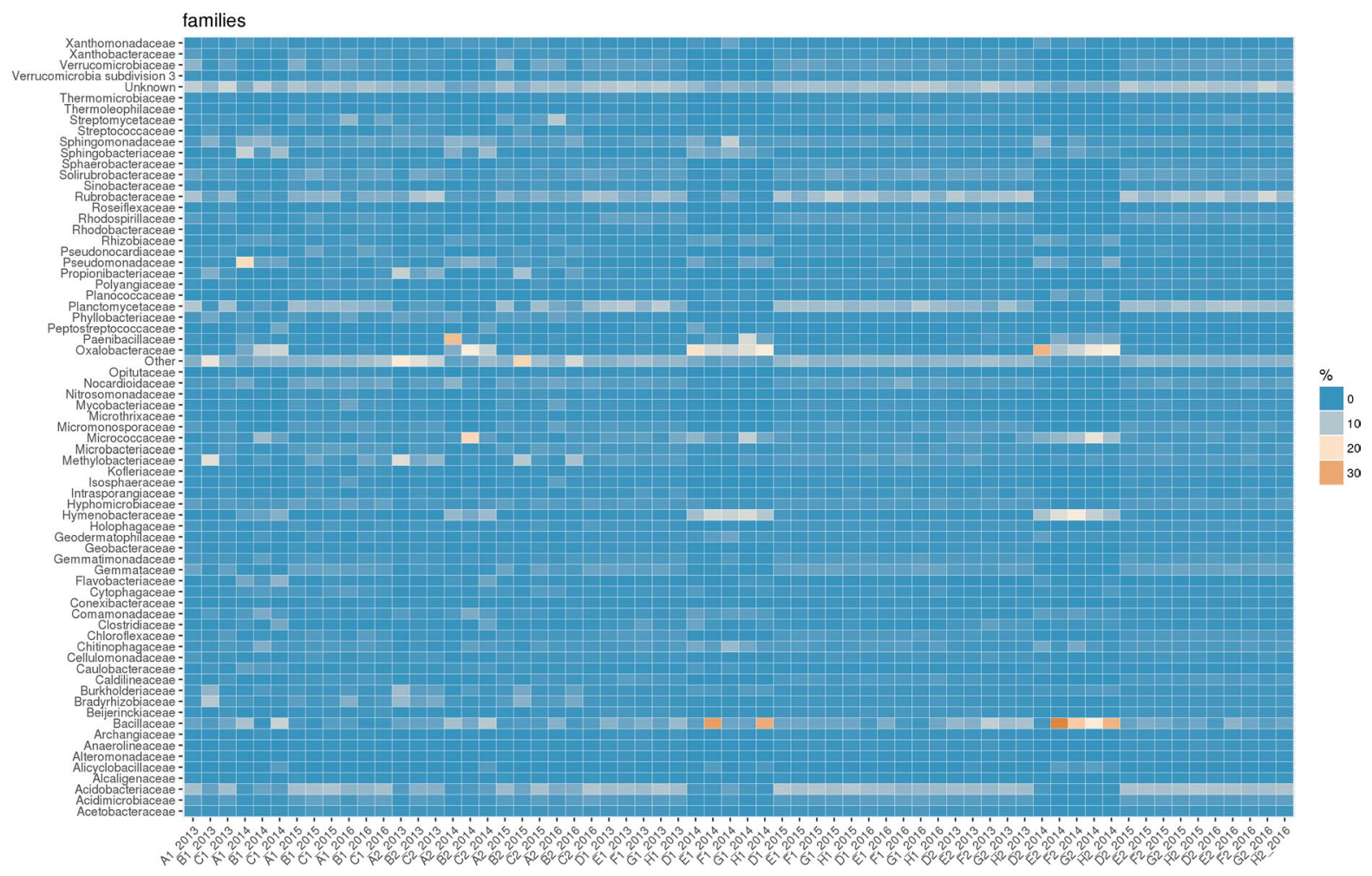

Fig. 4 Heatmap for data visualisation at the taxonomic level of family; abundance blocks of different taxa in the sampling sites during the 3 years following the flowline's construction

The relative abundances expressed in terms of $\log \mathrm{FC}$ covered a fairly wide range $(-6.06 \leq \log \mathrm{FC} \geq$ 7.88). The families with the most differential relative abundance are Psychromonadaceae, Neisseriaceae, Propionibacteriaceae, Leptotrichiaceae, Sphagnaceae and Pasteurellaceae.

As the comparison forest area 2015 vs forest area 2013, the assessment of identified taxa abundance shown in PCA presented a lower variability between 2013 and 2015 than the highlighted for the year 2014 in Fig. S3.

At the phylum level classification, there were no changes in the abundance of the 56 taxa identified at this taxonomic level. Of the 485 families detected, only nine were differentially abundant and in particular 7 (especially Cyclobacteriaceae and Cellvibrionaceae) over-represented and 2 (Brevibacteriaceae and Bacillaceae) under-represented, with variable fold changes in logarithmic scale between -3.16 and 4.66 (Table 1).
Comparing the forest area 2016 to forest area 2013, the PCA does not significantly distance biological diversity at the phylum level. A greater biological diversity exists within the forest area in 2013, primarily for site B at depth 0-20 cm (Fig. S4a and b).

A total of 485 taxonomic families were identified, of which 20 are significantly more rich (such as Microcoleaceae, Pinaceae and Williamsiaceae) and 16 less abundant (such as Psychromonadaceae and Leuconostocaceae) in 2016 compared to the reference year, with variable fold changes between -6.48 and 6.45 on a logarithmic scale (Table 1).

Comparing the microbial composition of samples collected in the cultivated area during 2014 to the area sampled in the 2013, at the phylum level, PCA showed a substantial variability among the samples of 2014, especially along the principal component 1 , which explains more than $87 \%$ of variability, while the samples taken in 2013 clustered together, due to a minimal variability (Fig. S5a). These samples 


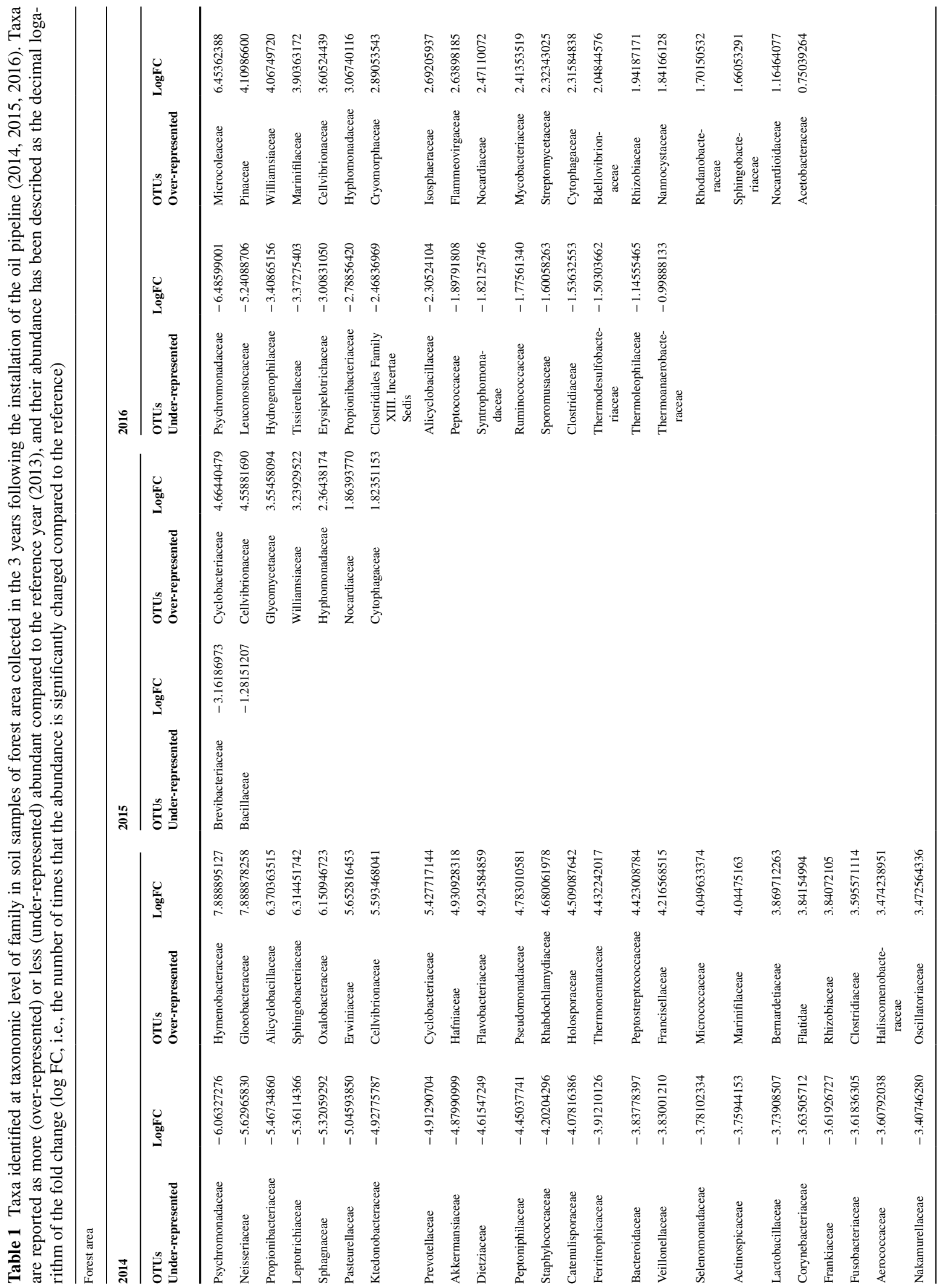




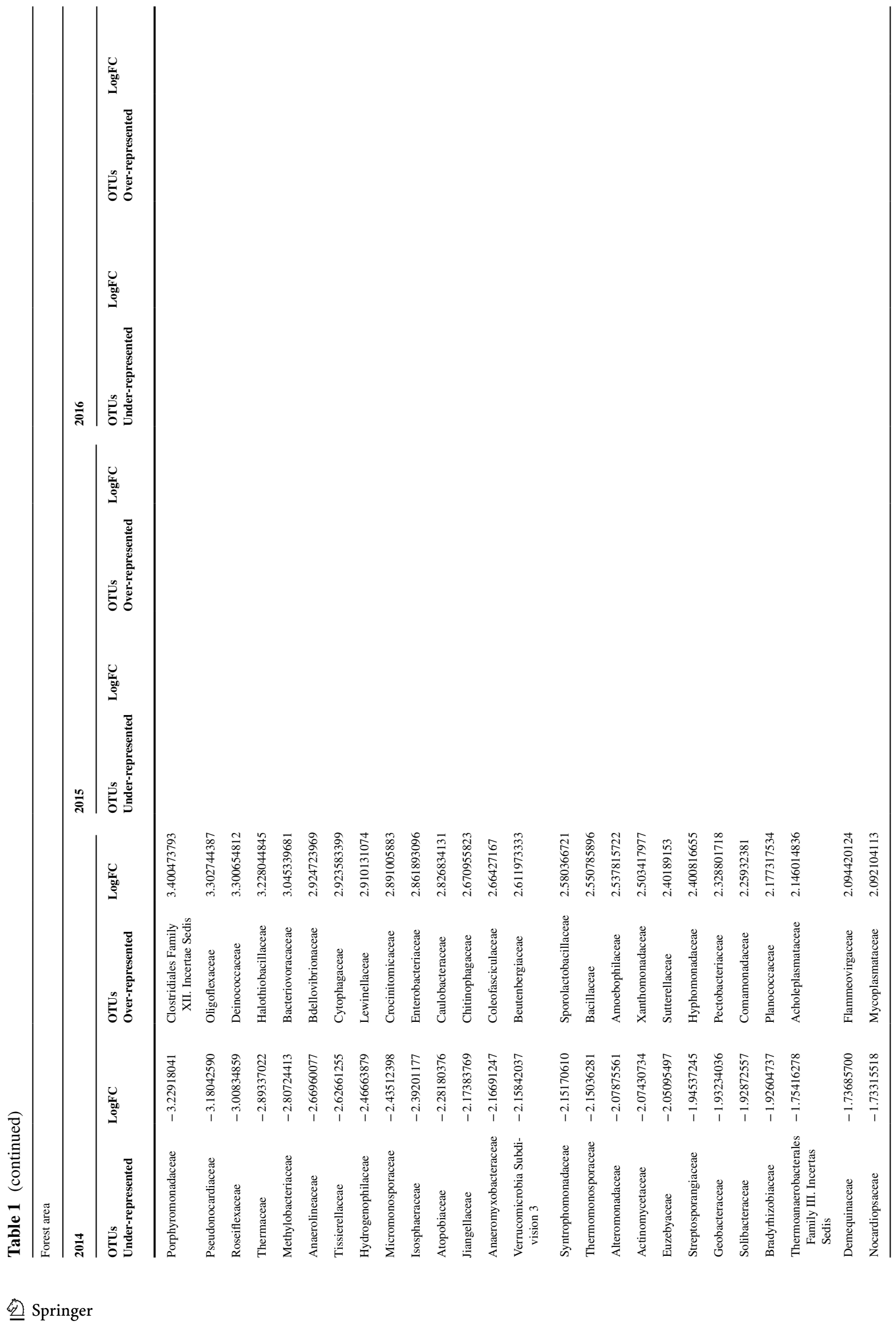




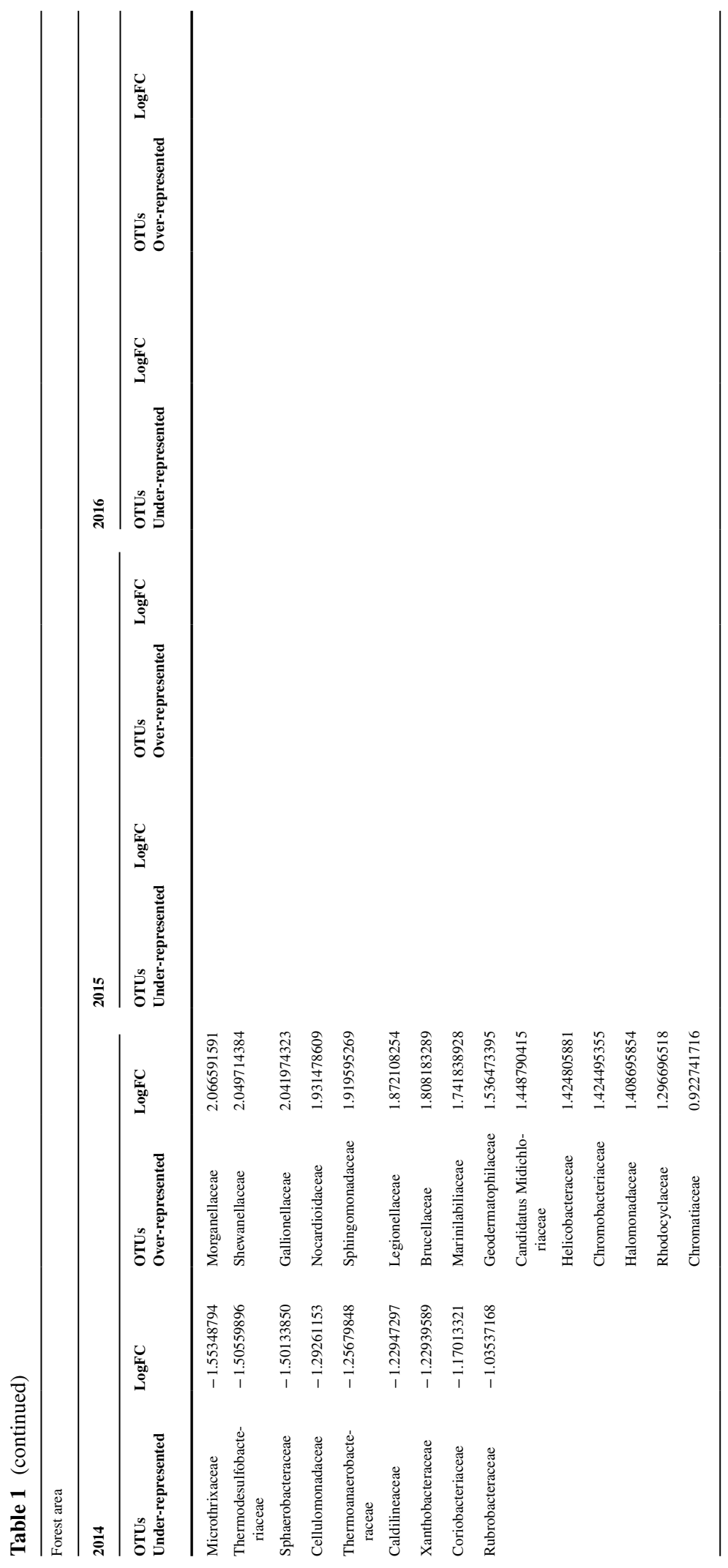


identified 56 phyla, of which 33 showed differential abundance in 2014; in particular, 14 are more abundant and 19 less abundant in 2014. At the taxonomic family level, the PCA still showed more significant variability among the 2014 samples (Fig. S5b).

The families identified were 485 , but only 260 were differentially abundant in 2014, 123 overrepresented and 137 under-represented (Table 2). Table 2 shows the identification of these taxa and their relative abundance.

The families most abundant were Flatidae, Hymenobacteraceae and Oxalobacteraceae, and those less copious were Ktedonobacteraceae, Methanosarcinaceae and Nitrososphaeraceae.

Comparing the microbial composition of samples collected in the cultivated area during 2015 to the area sampled in 2013, PCA showed a lower variability in 2015 than the reference year at the phylum taxonomic level. $X$-axis of Fig. S6a represents the main component 1 that explains $64 \%$ of the variability. Among the 56 phyla identified, 14 appear to be significantly plentiful in 2015 and 19 scarcely represented.

At the taxonomic family level, the PCA showed a heterogeneous variability in 2015 samples (Fig. S6b). The method used identified 485 families, of which 29 were significantly copious and 139 less plentiful in 2015, with fold changes ranging between -5.67 and 4.01 (Table 2). The families with the most differential relative abundance are Ktedonobacteraceae, Methanosarcinaceae, Nitrososphaeraceae and Williamsiaceae.

The biodiversity was evaluated at the phylum taxonomic level also comparing the cultivated area 2016 vs 2013. PCA showed a reasonable degree of variability among samples collected both in 2016 (along the principal component 2 that explains $32 \%$ of the total variability) and in 2013 (along with the main component 1 that sums up $52 \%$ of the variability) (Fig. S7a). The comparative analysis proved that 33 of the 56 identified phyla showed a significantly different abundance, as 14 were more present and 19 were in reduced quantity in the year 2016.

At the taxonomic family level, the PCA showed a high degree of diversity for the samples of 2013 and those of 2016 (Fig. S7b). The method recognised 485 families, of which 81 with significantly increased abundance (especially Stephanopyxidaceae, Coccomyxaceae, Fragilariaceae, Williamsiaceae and Chattonellaceae), and 63 with reduced quantity (in particular Methanosarcinaceae, Erysipelotrichaceae, Defluviitaleaceae and Prolixibacteraceae) in 2016 compared to 2013, with $\operatorname{logFC}$ between -5.31 and 5.38 (Table 2).

The differential analysis identified the taxa associated with the sampled soils' biological diversity, highlighting significant differences over time, especially comparing the initial situation (2013) to the first year of monitoring (2014). In 2014, we can appreciate the most significant perturbation of biodiversity. The comparative analysis showed taxa significantly different in the sampling areas, especially for the taxonomic levels of phylum and family.

The PCA results indicate a lower biological distance among the sampling points analysed in 2015 and 2016 compared to the reference year 2013, suggesting a certain degree of soil recovery.

The construction of the flowline caused changes in the bacterial community structure and their metabolic activity, with the appearance of different groups of bacteria. The analysis of 2014 sampling led to identifying bacteria commonly present in the soil where they mediate the complex metabolic processes and play a fundamental role in the functioning and stability of the system ensured by their diversification. Interesting was identifying bacterial groups naturally present in stressed soils such as bacteria belonging to Micrococcaceae, Xanthomonadaceae, Sporolactobacillaceae and Flavobacteriaceae families.

Bacterial groups resisting metals like cadmium, cobalt, zinc, chromium and mercury, or that reduce and detoxify redox-active metals, such as chromium and mercury, were in the identification list. Moreover, it was possible to identify bacterial groups belonging to the Pseudomonadaceae family, known to degrade particular harmful substances, such as naphthalene, toluene and other hydrocarbons. These beneficial bacteria are also able to degrade plastics and polystyrene to small substances.

Numerous studies have analysed soil sites contaminated with different pollutants in the past years, indicating diverse microbial populations present despite some extreme contamination conditions, showing as soils contaminated with heavy metals and hydrocarbons have undergone changes in community composition (Joynt et al., 2006; Banerjee et al., 2011). The difference in soils' source and properties has provided different bacterial isolates, such as Acinetobacter, Aeromonas, Aureobacterium, Bacillus, Escherichia, 
Klebsiella, Micrococcus, Pseudomonas, Rhodococcus and Stenotrophomonas (Anderson \& Cook, 2004; Jackson et al., 2005; Aksornchu et al., 2008; Vinas et al., 2005). Mathè et al. (2012) revealed how different pollutants affect the activity and diversity of endogenous microbiota. Individual isolates were tested for their ability to degrade various types of hydrocarbons (aliphatic-, mono-aromatic and polycyclic aromatic hydrocarbons) or for their capability to resist heavy metals or to increase in the presence of antibiotics. Results revealed that in contaminated sites, an increased activity of hydrocarbonoclastic bacteria occurs, supported by significant $\mathrm{CO}_{2}$ production. Furthermore, Alisi et al. (2009) and Sprocati et al. (2012) showed the feasibility of the remediation of a soil containing heavy metals and spiked with diesel fuel, through a bioaugmentation strategy based on the use of a microbial formula tailored to selected natives, which can effectively facilitate and speed up the bioremediation of matrices co-contaminated with hydrocarbons and heavy metals.

During the monitoring analyses in 2014, we observed an impressive presence of specific bacterial groups already found dominant in soil taken directly from oil wells (Galazka et al., 2018). Some families of Alphaproteobacteria, Rhizobiaceae, Rhodobacteraceae, Acetobacteraceae and Sphingomonadaceae were present in these samples. Bacteria that use hydrocarbons as the only carbon source were in the list and belonged to Vibrionaceae, Bacillaceae, Moraxellaceae, Mycobacteriaceae, Sphingomonadaceae, Nocardiaceae and Flavobacteriaceae families (Zhong et al., 2011). Moreover, Bacillaceae, Rhizobiaceae, Nocardiaceae, Actinomycetaceae, Nocardiaceae and Streptomycetaceae bacterial groups, associated with hydrocarbon degradation (Zhong et al., 2011; Doong \& Lei, 2003; Sutton et al., 2013; Naether et al., 2012), occurred.

We also observed a differential abundance for Methylobacteriaceae, which grew on methanol and other one-carbon compounds as sources of energy and carbon, and Geobacteraceae, including several species that oxidise monoaromatic hydrocarbons, such as toluene and benzene. Then, the family Methanosarcinaceae, with the methanogenic Archaea, is most adaptable to different substrates used for energy generation and found in a wide variety of anaerobic producing methane environments (water streams, marine and hypersaline sediments, wetlands, thermal habitats, oil wells, anaerobic waste treatment systems and gastrointestinal tracts of animals) (Wegner \& Liesack, 2016; Msaddak et al., 2017; Sitte et al., 2010). The presence and rapid growth of particular bacterial groups in the analysed sites indicate a natural bioremediation process to restore optimal conditions of soils altered by the flowline construction and oil pipeline presence in the underground.

\section{Conclusions}

In conclusion, this exciting research activity needs a more in-depth investigation to understand soil bacterial populations' activities and dynamics and the relationships between functionality and microbial diversity that underlie significant recovery and restoration processes. Although many species are necessary for the maintenance of stable processes in ever-changing ecosystems, a minimum number of species exerting a specific ecological role are essential. The greater the degree of intra- or inter-specific functional biodiversity of an ecosystem, the greater is its tolerance to perturbations and its resilience, since there will be more chances that genotypes or species can replace the functions of those disappeared.

On September 2015, a cross-sector guide for implementing the mitigation hierarchy was ready for dissemination by the Biodiversity Consultancy on behalf of International Petroleum Industry Environmental Conservation Association (IPIECA), the International Council Mining and Metals (ICMM) and the Equator Principles Association. The CrossSector Biodiversity Initiative (CSBI) makes available to people the Mitigation Hierarchy Guide and an executive summary, http://www.csbi.org.uk/ our-work/mitigation-hierarchy-guide/ (visited on 30 December 2020).

The mission of CSBI is "developing and sharing good practices related to biodiversity and ecosystem services in the extractive industries".

The mitigation hierarchy is an instrument designed to help users reduce, as much as possible, the negative impacts of development projects on biodiversity and ecosystem services (BES).

It includes four crucial actions - "avoid", "minimise", "restore" and "compensate" — and provides a best practice approach to assist in the sustainable management of natural and living resources by 


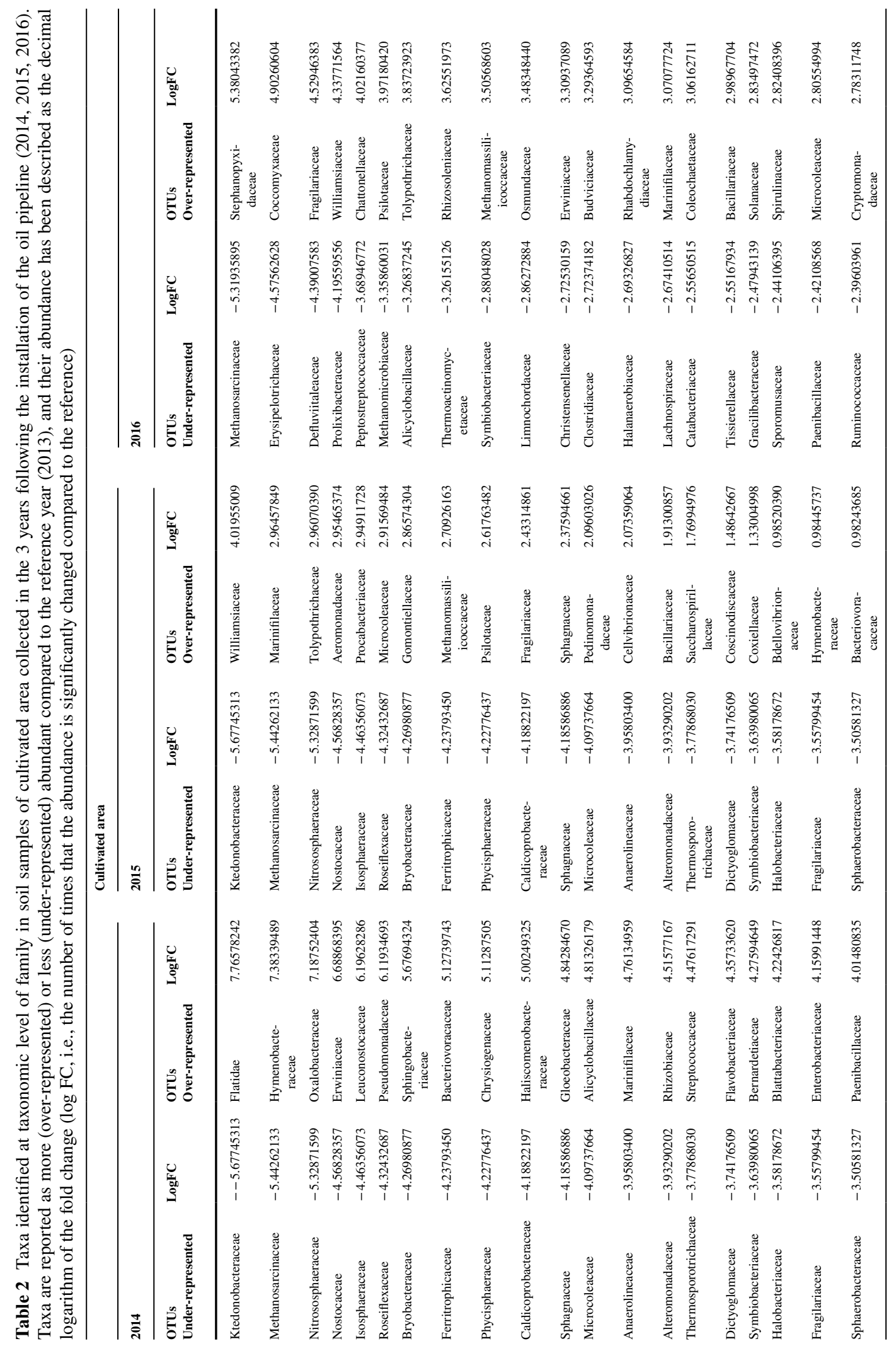




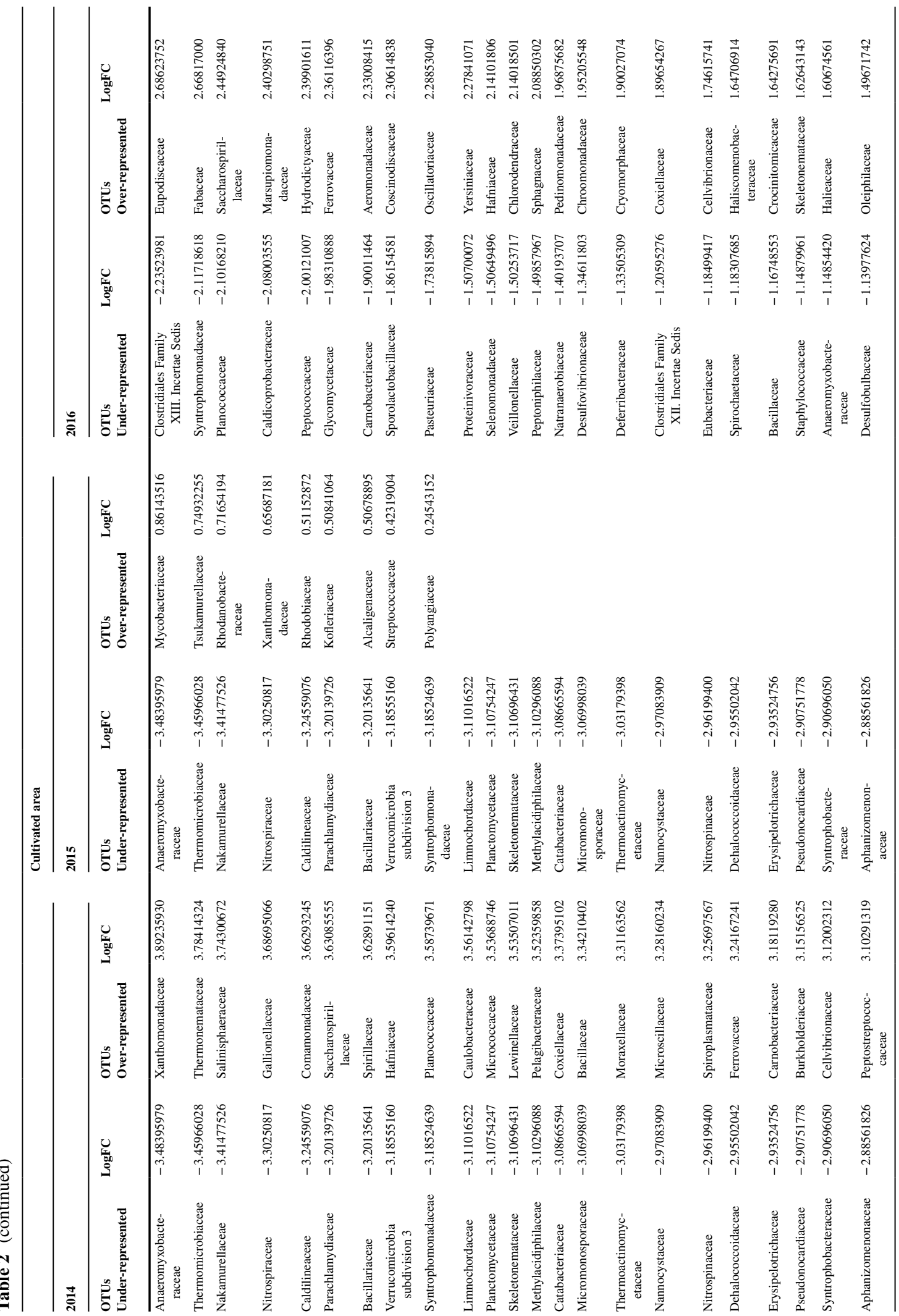




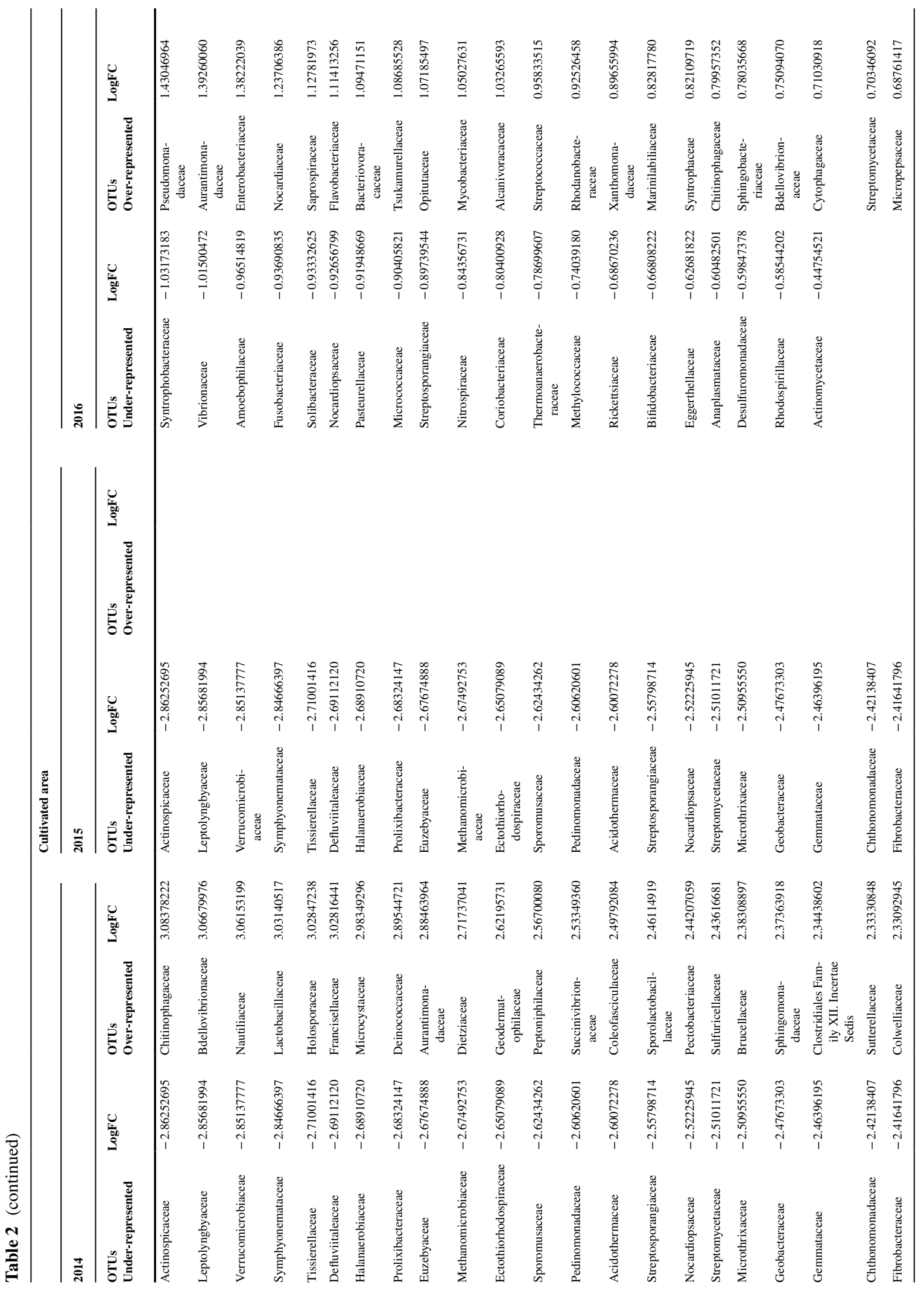




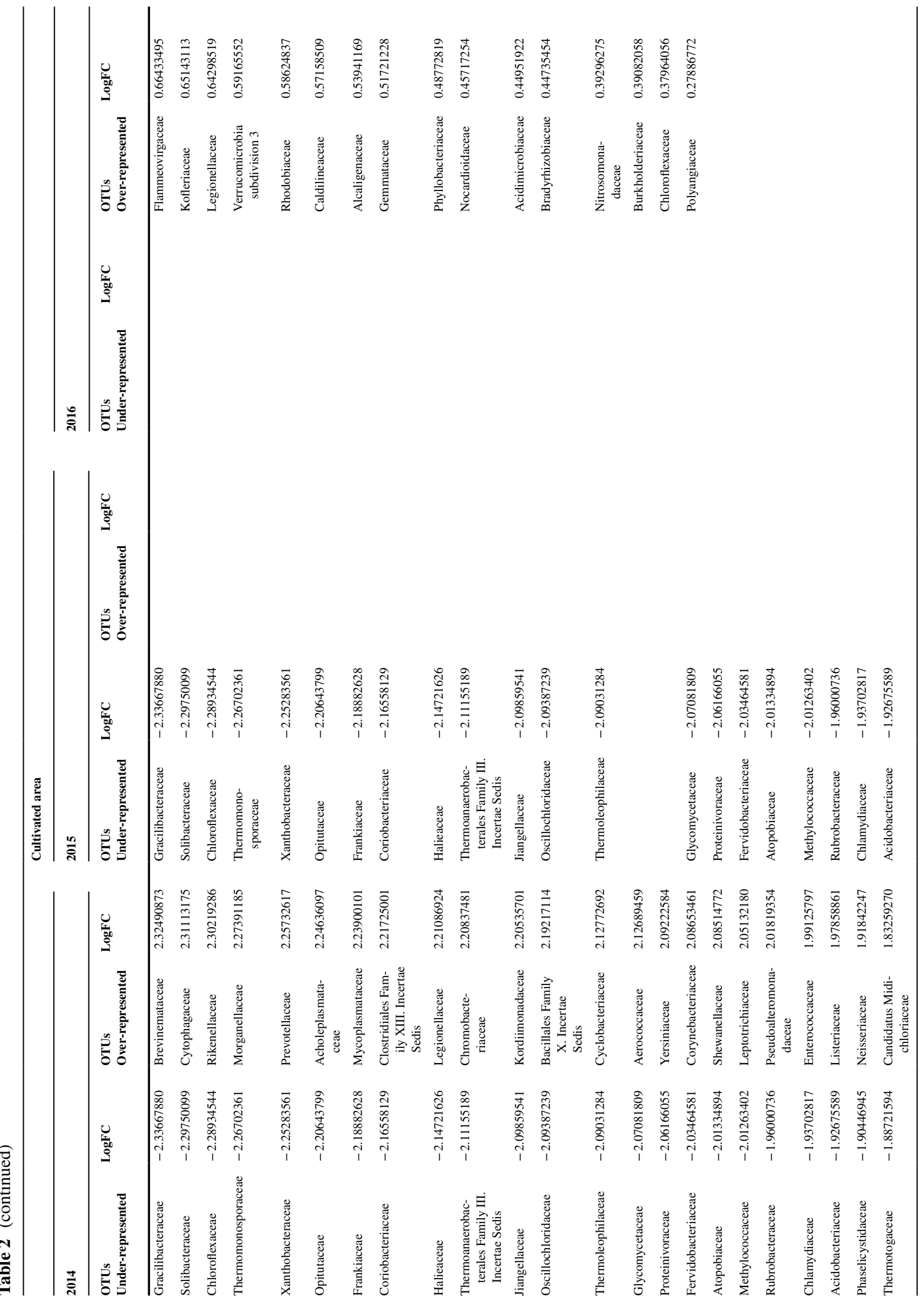




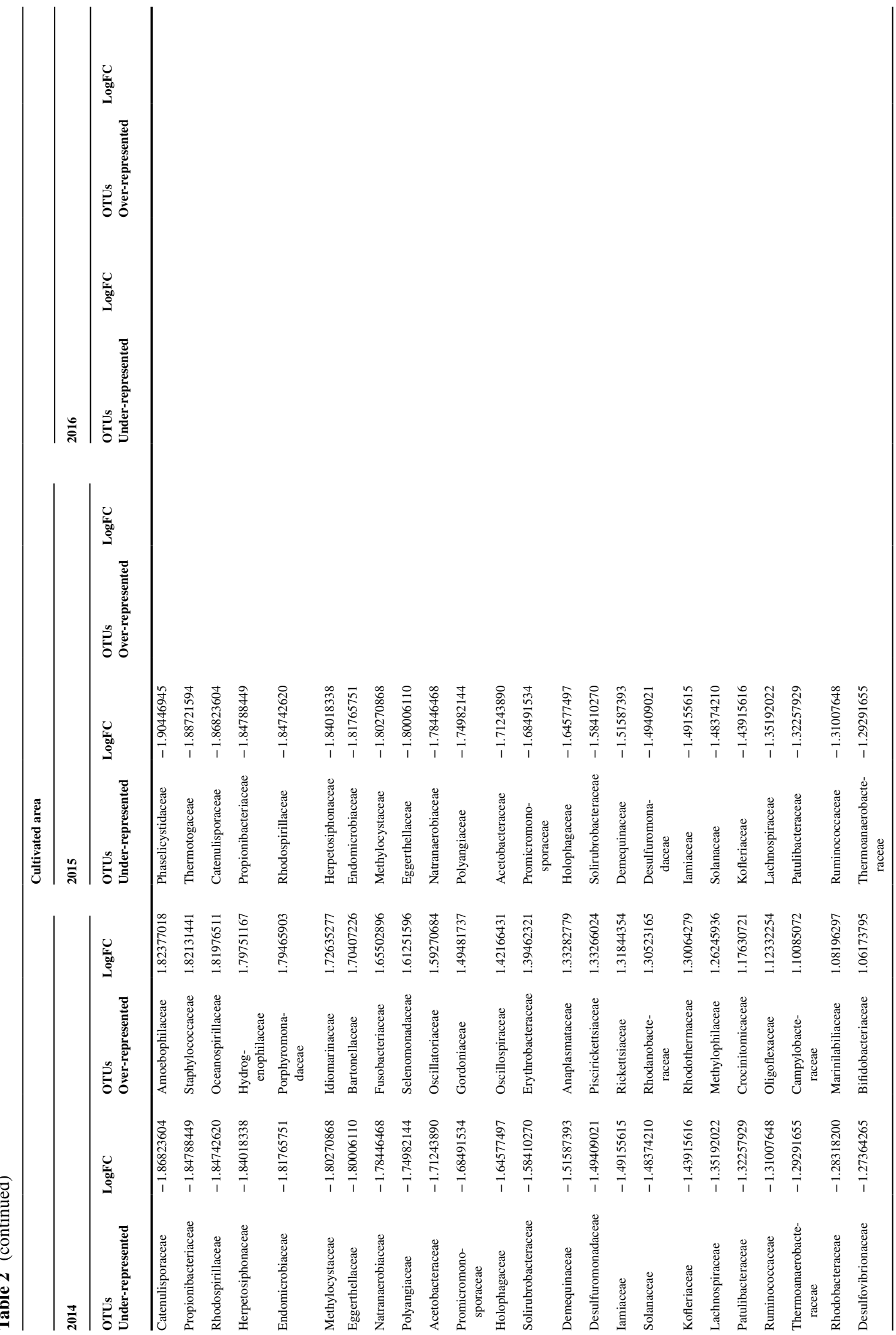




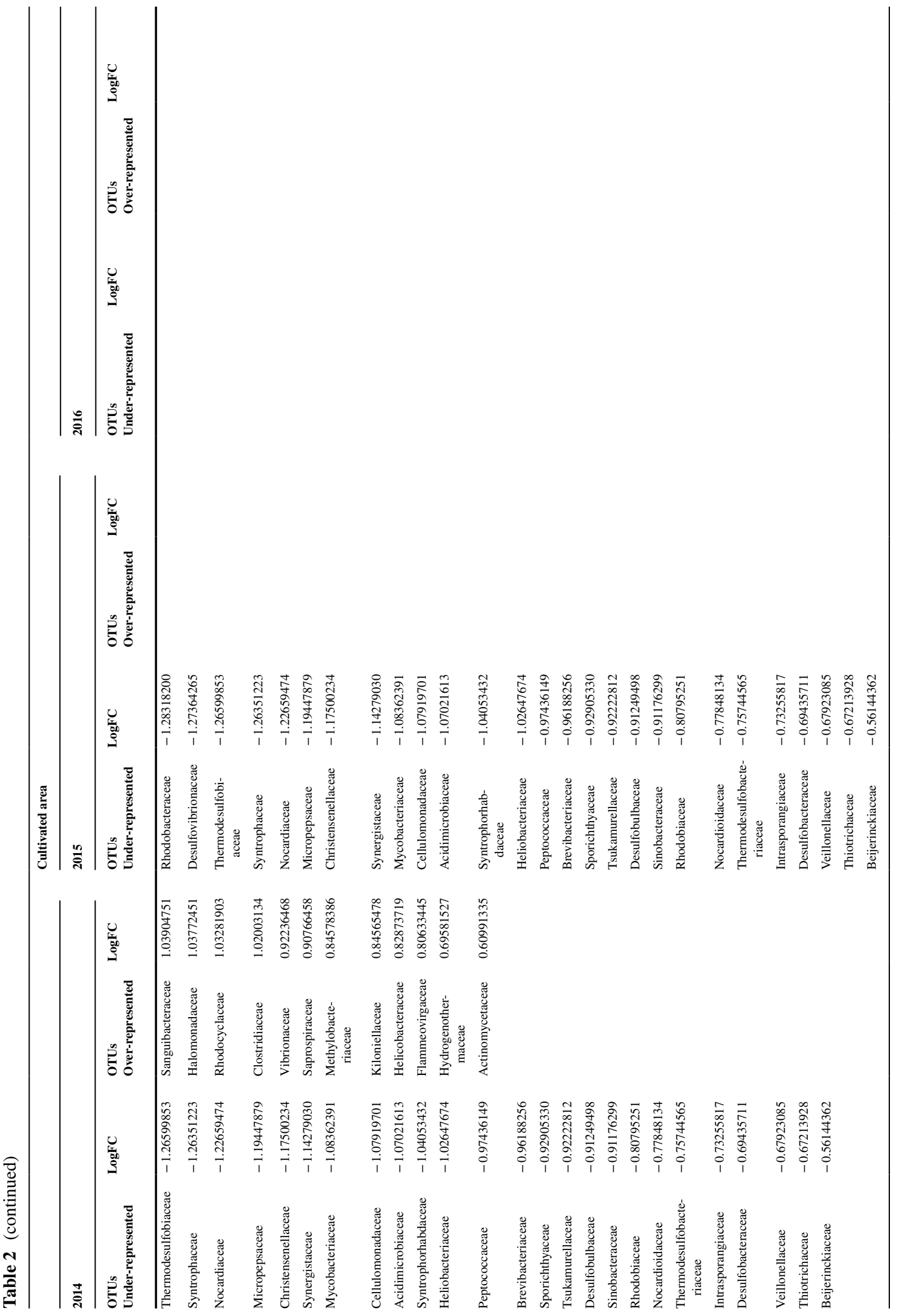


establishing a mechanism to balance the conservation needs with priority development. So, the monitoring work reported in this article can be considered one of the actions necessary to verify the restoration of natural equilibrium in an area that has suffered an anthropic insult.

Author contribution Conceptualization, EB, GS and SAB; methodology, MGB and LS; DNA extraction and bioinformatic analysis K.DT.; validation, MGB, GS and SAB; formal analysis, MGB and LC; initial draft preparation, MGB; review and editing, MGB, GS and SAB.; supervision, GS.; funding acquisition and flowline construction, EB. All authors have read and agreed to the published version of the manuscript.

Funding The work of monitoring activity was supported by SAIPEM — S.p.A., private company.

Availability of data and material Not applicable.

Code availability Not applicable.

\section{Declarations}

Conflict of interest The authors declare no competing interests.

Open Access This article is licensed under a Creative Commons Attribution 4.0 International License, which permits use, sharing, adaptation, distribution and reproduction in any medium or format, as long as you give appropriate credit to the original author(s) and the source, provide a link to the Creative Commons licence, and indicate if changes were made. The images or other third party material in this article are included in the article's Creative Commons licence, unless indicated otherwise in a credit line to the material. If material is not included in the article's Creative Commons licence and your intended use is not permitted by statutory regulation or exceeds the permitted use, you will need to obtain permission directly from the copyright holder. To view a copy of this licence, visit http://creativecommons.org/licenses/by/4.0/.

\section{References}

Ahmed, V., Verma, M. K., Gupta, S., Mandhan, V., \& Chauhan, N. S. (2018). Metagenomic profiling of soil microbes to mine salt stress tolerance genes. Frontiers in Microbiology, $9,1-11$.
Aksornchu, P., Prasertsan, P., \& Sobhon, V. (2008). Isolation of arsenic-tolerant bacteria from arsenic-contaminated soil. Songklanakarin Journal of Science and Technology, 30, 95-102.

Alisi, C., Musella, R., Tasso, F., Ubaldi, C., Manzo, S., Cremisini, C., \& Sprocati, A. R. (2009). Bioremediation of diesel oil in a co-contaminated soil by bioaugmentation with a microbial formula tailored with native strains selected for heavy metals resistance. Science of the Total Environment, 407, 3024-3032.

Anderson, C. R., \& Cook, G. M. (2004). Isolation and characterisation of arsenate-reducing bacteria from arsenic contaminated sites in New Zealand. Current Microbiology, 48, 341-347.

Arias, M. E., Gonzalez-Perez, J. A., Gonzalez-Vila, F. J., \& Ball, A. S. (2005). Soil health-a new challenge for microbiologists and chemists. International Microbiology, 8, 13-21.

Banerjee, S., Datta, S., Chattyopadhyay, D., \& Sarkar, P. (2011). Arsenic accumulating and transforming bacteria isolated from contaminated soil for potential use in bioremediation. Journal of Environmental Science and Health, 46(14), 1736-1747.

Bloem, J., Benedetti, A., Hopkins D. (2006). Microbial methods assessing soil quality CABI Publishing 0-85199-098-3.

Bonomo, M. G., Cafaro, C., Guerrieri, G., Crispo, F., Milella, L., Calabrone, L., \& Salzano, G. (2017). Flow cytometry and capillary electrophoresis analyses in ethanol-stressed Oenococcus oeni strains and changes assessment of membrane fatty acids composition. Journal of Applied Microbiology, 122(6), 1615-1626.

Bonomo, M. G., \& Salzano, G. (2013). Genotypic and technological diversity of Leuconostoc mesenteroides and Lactobacillus paracasei strains for use as adjunct starter cultures in 'Pecorino di Filiano' cheese. International Journal of Dairy Technology, 66(3), 402-409.

Bonomo, M. G., Milella, L., Martelli, G., \& Salzano, G. (2013). Stress response assessment of Lactobacillus sakei strains selected as potential autochthonous starter cultures by flow cytometry and nucleic acid double staining analyses. Journal of Applied Microbiology, 115(3), 786-795.

Cafaro, C., Bonomo, M. G., Guerrieri, A., Crispo, F., Ciriello, R., \& Salzano, G. (2016). Assessment of the genetic polymorphism and physiological characterisation of indigenous Oenococcus oeni strains isolated from Aglianico del Vulture red wine. Folia Microbiolica, 61, 1-10.

Carbonetto, B., Rascovan, N., Alvarez, R., Mentaberry, A., \& Vazquez, M. P. (2014). Structure, composition and metagenomic profile of soil microbiomes associated to agricultural land use and tillage systems in Argentine Pampas. PLoS One, 9 (6), e99949.

Daniel, R. (2005). The metagenomics of soil. Nature Reviews Microbiology, 3, 470-478.

Doong, R. A., \& Lei, W. G. (2003). Solubilization and mineralisation of polycyclic aromatic hydrocarbons by Pseudomonas putida in the presence of surfactant. Journal of Hazardous Materials, 96, 15-27.

Gałazka, A., Grzadziel, J., Gałazka, R., Ukalska-Jaruga, A., Strzelecka, J., \& Smreczak, B. (2018). Genetic and functional diversity of bacterial microbiome in soils with 
long term impacts of petroleum hydrocarbons. Frontiers Microbiology, 9, 1923.

Gomez, E., Garland, J., \& Conti, M. (2004). Reproducibility in the response of soil bacterial community-level physiological profiles from a land use intensification gradient. Applied Soil Ecology, 26, 21-30.

Kirk, J. L., Beaudette, L. A., Hart, M., Moutoglis, P., Klironomos, J. N., Lee, H., \& Trevors, J. T. (2004). Methods of studying soil microbial diversity. Journal of Microbiological Methods, 58, 169-188.

Klimek, B., Sitarz, A., Choczyński, M., \& Niklińska, M. (2016). The effects of heavy metals and total petroleum hydrocarbons on soil bacterial activity and functional diversity in the Upper Silesia Industrial Region (Poland). Water Air Soil Pollution, 227, 265.

Jackson, C. R., Dugas, S. L., \& Harrison, K. G. (2005). Enumeration and characterisation of arsenate-resistant bacteria in arsenic free soils. Soil Biology and Biochemistry, 37, 2319-2322.

Joynt, J., Bischoff, M., Turco, R., Konopka, A., \& Nakatsu, C. H. (2006). Microbial community analysis of soils contaminated with lead, chromium and petroleum hydrocarbons. Microbial Ecology, 51, 209-219.

Lelario, F., Scrano, L., De Franchi, S., Bonomo, M. G., Salzano, G., Milan, S., Milella, L., \& Bufo, S. A. (2018). Identification and antimicrobial activity of most representative secondary metabolites from different plant species. Chemical and Biological Technologies in Agriculture, 5, 13.

Liu, Q., Tang, J., Gao, K., Gurav, R., \& Giesy, J. P. (2017). Aerobic degradation of crude oil by microorganisms in soils from four geographic regions of China. Scientific Reports, 7, 14856.

Mader, P., Fliebach, A., Dubois, D., Gunst, L., Fried, P., \& Niggli, U. (2002). Soil fertility and biodiversity in organic farming. Science, 296(5573), 1694-1697.

Malla, M. A., Dubey, A., Yadav, S., Kumar, A., Hashem, A., \& Abd Allah, E. F. (2018). Understanding and designing the strategies for the microbe-mediated remediation of environmental contaminants using omics approaches. Frontiers MicrobiolOgy, 9, 1132.

Máthé, I., Benedek, T., Táncsics, A., Palatinszky, M., Lányi, S., \& Márialigeti, K. (2012). Diversity, activity, antibiotic and heavy metal resistance of bacteria from petroleum hydrocarbon contaminated soils located in Harghita County (Romania). International Biodeterioration \& Biodegradation, 73, 41-49.

Mocali, S., \& Benedetti, A. (2010). Exploring research frontiers in microbiology: The challenge of metagenomics in soil microbiology. Research in Microbiology, 161, 497-505.

Msaddak, A., Durán, D., Rejili, M., Mars, M., Ruiz-Argüeso, T., Imperial, J., Palacios, J., \& Rey, L. (2017). Diverse bacteria affiliated with the genera Microvirga, Phyllobacterium, and Bradyrhizobium nodulate Lupinus micranthus growing in soils of Northern Tunisia. Applied Environmental Microbiology, 2, 83(6).

Naether, A., Foesel, B. U., Naegele, V., Wüst, P. K., Weinert, J., \& Bonkowski, M. (2012). Environmental factors affect acidobacterial communities below the subgroup level in grassland and forest soils. Applied Environmental Microbiology, 78, 7398-7406.

Peng, M., Zi, X., \& Wang, Q. (2015). Bacterial community diversity of oil contaminated soils assessed by high throughput sequencing of 16S rRNA genes. International Journal of Environmental Research and Public Health, 12, 12002-12015.

Pichler, M., Coskun, Ö. K., Ortega-Arbulú, A. S., Conci, N., Wörheide, G., Vargas, S., \& Orsi, W. D. (2018). A 16S rRNA gene sequencing and analysis protocol for the Illumina MiniSeq platform. MicrobiologyOpen, 7, e611.

Rutgers, M., Wouterse, M., Drost, S. M., Breure, A. M., Mulder, C., Stone, D., Winding, A., \& Bloem, J. (2016). Monitoring soil bacteria with community-level physiological profiles using BiologTM ECO-plates in the Netherlands and Europe. Applied Soil Ecology, 97, 23-35.

Schloss, P. D., \& Handelman, J. (2005). Metagenomics for studying unculturable microorganisms: Cutting the Gordian knot. Genome Biology, 6(8), 229.

Sitte, J., Akob, D. M., Kaufmann, C., Finster, K., Banerjee, D., Burkhardt, E. M., Kostka, J. E., Scheinost, A. C., Büchel, G., \& Küsel, K. (2010). Microbial links between sulfate reduction and metal retention in uranium- and heavy metal-contaminated soil. Applied Environmental Microbiology, 76(10), 3143-3152.

Sofo, A., Elshafie, H. S., Scopa, A., Mang, S. M., \& Camele, I. (2018). Impact of airborne zinc pollution on the antimicrobial activity of olive oil and the microbial metabolic profiles of $\mathrm{Zn}$-contaminated soils in an Italian olive orchard. Journal of Trace Elements in Medicine and Biology, 49, 276-284.

Sorensen, J., Nicolaisen, M. H., Ron, E., \& Simonet, P. (2009). Molecular tools in rhizosphere microbiology-from singlecell to whole-community analysis. Plant and Soil, 321, 483-512.

Sprocati, A. R., Alisi, C., Tasso, F., Marconi, P., Sciullo, A., Pinto, V., Chiavarini, S., Ubaldi, C., \& Cremisini, C. (2012). Effectiveness of a microbial formula, as a bioaugmentation agent, tailored for bioremediation of diesel oil and heavy metal co-contaminated soil. Process Biochemistry, 47, 1649-1655.

Sutton, N. B., Maphosa, F., Morillo, J., Abu Al-Soud, W., Langenhoff, A. A. M., \& Grotenhuis, T. (2013). Impact of long-term diesel contamination on soil microbial community structure. Applied Environmental Microbiology, 79, 619-630.

Torsvik, V., \& Ovreas, L. (2002). Microbial diversity and function in soil: From genes to ecosystems. Current Opinion in Microbiology, 5, 240-245.

van Elsas, J. D., Jansson, J. K., \& Trevors, J. T. (2007). Modern soil microbiology. CRC Press.

Vinas, M., Sabaté, J., Espuny M. J., \& Solanas, A. M. (2005). Bacterial community dynamics and polycyclic aromatic hydrocarbon degradation during bioremediation of heavily creosote-contaminated soil. Applied Environmental Microbiology, 7008-7018.

Wegner, C. E., \& Liesack, W. (2016). Microbial community dynamics during the early stages of plant polymer breakdown in paddy soil. Environmental Microbiology, 18(9), 2825-2842.

Zhong, Y., Luan, T., Lin, L., Liu, H., \& Tam, N. F. Y. (2011). Production of metabolites in the biodegradation of phenanthrene, fluoranthene and pyrene by the mixed culture of Mycobacterium sp. and Sphingomonas sp. Bioresource Technology, 102, 2965-2972. 
Publisher's Note Springer Nature remains neutral with regard to jurisdictional claims in published maps and institutional affiliations. 